\title{
Erratum to: The Commonalities of Global Crises: Markets, Communities and Nostalgia
}

Christian Karner • Bernhard Weicht

DOI 10.1057/978-1-137-50273-5

Author affiliation in: Copyright page, First contributor-Christian Karner

Christian Karner

University of Nottingham

Mühlheim, Germany

Changed as

Christian Karner

University of Nottingham

Nottingham, United Kingdom

The online version of the updated original book can be found under DOI 10.1057/978-1-137-50273-5

(C) The Editor(s) (if applicable) and The Author(s) 2016

C. Karner, B. Weicht (eds.), The Commonalities of Global Crises, DOI 10.1057/978-1-137-50273-5_14 
Christian Karner

University of Nottingham

Nottingham, United Kingdom

Bernhard Weicht

Department of Sociology,

University of Innsbruck

Innsbruck, Austria 